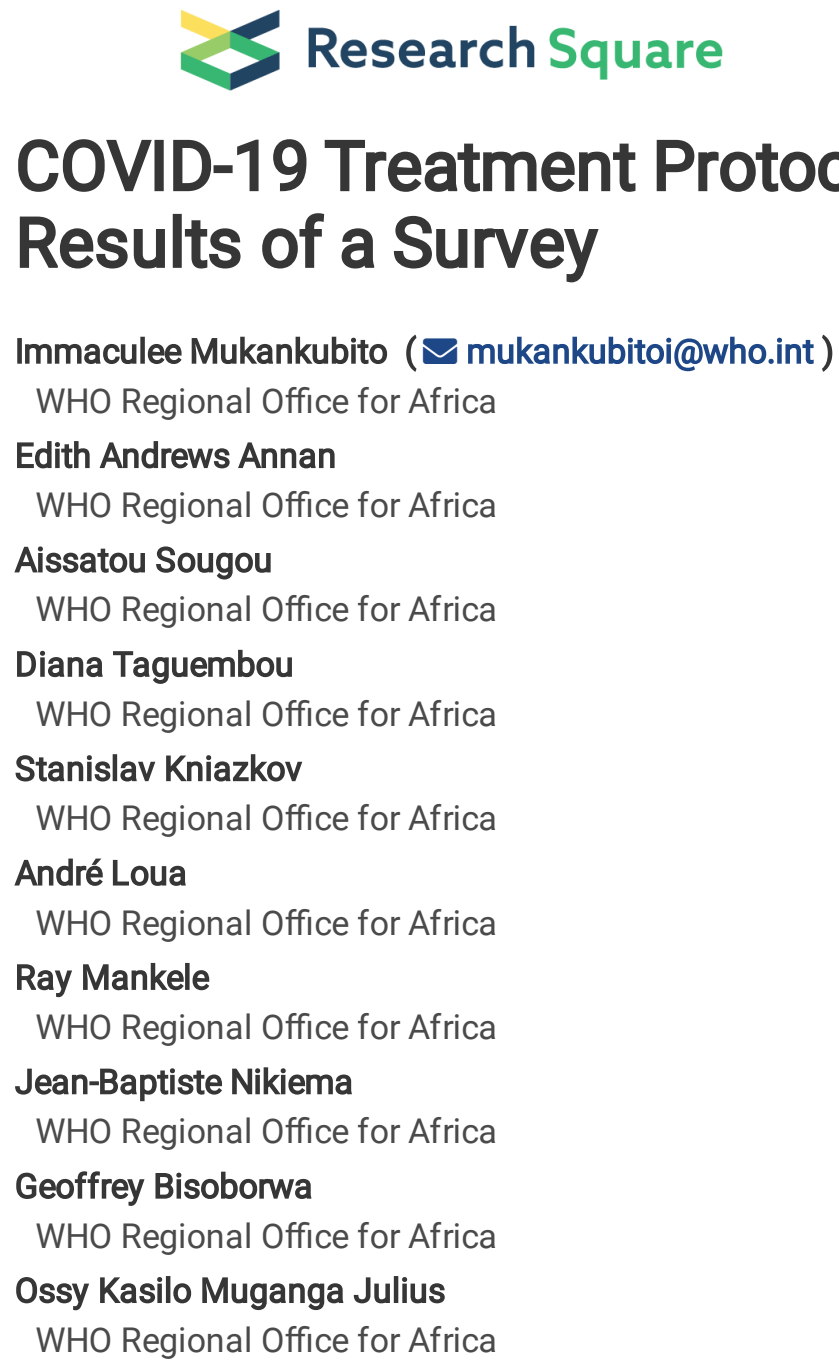

\title{
COVID-19 Treatment Protocols in the WHO African Region - Results of a Survey
}

\section{Research Article}

Keywords: Africa, COVID-19, Treatment, Guidelines, Protocols, Therapeutics, AMR

Posted Date: May 14th, 2021

DOI: https://doi.org/10.21203/rs.3.rs-519255/v1

License: (c) (i) This work is licensed under a Creative Commons Attribution 4.0 International License. Read Full License 


\section{Abstract}

Background: As of May 2021 no ethiotropic treatment for COVID-19 demonstrated safety and efficacy. A number of clinical trials are underway to investigate candidate products, most of them are repurposed products. As new evidenced emerges, WHO updates its recommendations on clinical management to support Member States amending their treatment protocols.

Hydroxychloroquine / Chloroquine (HCQ/CQ) and Lopinavir + Ritonavir arms of the Solidarity Trial were discontinued. There is strong recommendation against administrating these products to COVID-19 patients. In contrary, Corticosteroids were identified as lifesaving medicines, substantially reducing mortality for critically ill patients. This study aimed at checking the alignment of countries' COVID-19 treatment protocols in the WHO African region with the WHO recommendations, and analyze their impact on supply chain and quality of care.

Methods: In October-November 2020, country treatment protocols were collected, and data on therapeutics were summarized in an Excel Sheet to facilitate their comparison with the WHO recommendations.

Results: From the thirty protocols collected, 50\% were recommending HCQ/CQ for mild COVID-19. The proportion was quite similar for moderate and severe cases accounting $57 \%$ and $53 \%$ of protocols respectively, while $27 \%$ were still recommending HCQ/CQ for critically ill patients. Antibiotics were recommended in $53 \%$ and $80 \%$ of protocols for mild and moderate cases respectively. Only $47 \%$ and $60 \%$ of protocols were recommending corticosteroids for severe and critical cases respectively. Convalescent Plasma was reflected in few protocols.

Discussion: HCQ and CQ were the standard treatment of COVID-19 in most of countries, diverting resources from the supply chain for essential services. There is a serious risk of antibiotic misuse, which can potentially lead to antimicrobial resistance surge, the increased rates of morbidity, mortality, treatment costs and financial burden for patients and communities. Slow uptake of corticosteroids may compromise the quality of care for severe and critical cases.

Conclusions: The COVID-19 pandemic has demonstrated that, in cases similar to COVID-19 without efficacious treatment, treatment protocols are handled as living documents to be timely amended. Proactive update of country protocols to reflect evolving scientific evidences is critical, to ensure that all patients benefit from high standard quality of care.

\section{Background}

The first case of severe acute respiratory syndrome coronavirus-2 (SARS-CoV-2) that causes Coronavirus disease 2019 (COVID19) was reported in Wuhan, People Republic of China in December 2019. In January 2020 and March 2020, the World Health Organization (WHO) declared COVID-19 a Public Health Emergency of International Concern and a pandemic respectively. The First case in the WHO African region, was reported in Algeria on 25 February 2020 [1].

Global and regional mechanisms involving various networks of experts and partnerships, have been activated to provide a comprehensive technical package and guidance materials for the preparedness and response, which are updated and adapted regularly as the pandemic evolves [1, 2].

In January 2020, WHO activated its incident management support team (IMST), to ensure the coordination of activities and response across the three levels of WHO, as well as its research and development (R\&D) Blueprint to fast-track the availability of effectives diagnostics, vaccines and therapeutics for COVID-19 [1, 2].

In March 2020, WHO and partners launched the Solidarity trial, to generate robust data from around the world to find the most effective treatments for COVID-19. Later in April 2020, the Access to COVID-19 Tool Accelerator (ACT-A) partnership was launched, to support the fastest, most coordinated, and successful global effort in developing and availing essential tools to prevent, detect and treat COVID-19 [1].

Related to supplies, WHO launched the COVID-19 Supply Portal in May 2020, a purpose-built tool to facilitate and consolidate countries' requests of critical healthcare supplies. In July 2020, the COVAX facility, a key part of the Access to COVID-19 Tools Accelerator (ACT-A) mechanism was designed to guarantee rapid, fair and equitable access to COVID-19 vaccines worldwide. In 
September 2020, WHO issued a framework offering guidance on the allocation of COVID-19 vaccines between countries, and on the prioritization of groups for vaccination within countries as endorsed by the Strategic Advisory Group of Experts (SAGE) on immunization. The SAGE will continue to provide recommendations on the vaccine use based on robust scientific evidences in the context of COVID-19 [1].

To guide practices worldwide, WHO has published various documents about COVID-19. Of these, more than half are detailed technical guidance on several topics such as how: to prevent the disease to spread, trace, quarantine, test and treat the cases depending on the severity of the illness, to protect health care workers, and to help communities to respond appropriately [2,3]. The published WHO guidelines for clinical management of COVID-19 have been translated into protocols for treatment of COVID19 patients by some countries.

Although most infections are not severe, some patients with COVID-19 can quickly develop a critical and deadly disease, requiring admission to Intensive Care Unit (ICU). Following WHO recommendations, clinical and therapeutic management of COVID-19 is described by disease severity and mostly treatment is for preventing and controlling secondary infections, disease complications, treating symptoms, acute co-infections, underlying diseases, ensure the life sustaining and promptly support organs failure, including supplemental oxygen and mechanical ventilation support when required $[4,5]$.

For Mild COVID-19 without evidence of viral pneumonia or hypoxia accounting 40\% of global cases, and Moderate COVID-19 with clinical signs of pneumonia but no signs of severe pneumonia, including oxygen saturation (SpO2) $\geq 90 \%$ on room air representing $40 \%$ of global cases, WHO recommend symptomatic treatment such as antipyretics to alleviate fever and pain, and is against antibiotic therapy or prophylaxis unless there is a clinical suspicion of a bacterial infection [4].

Regarding Severe COVID-19 accounting $15 \%$ of global cases, defined by any of the following signs: oxygen saturation < $90 \%$ on room air; respiratory rate $>30$ breaths per minute in adults and raised in respiratory rate in children and signs of severe respiratory distress (SRD); WHO recommends supplemental oxygen therapy, cautious intravenous fluids management, antimicrobial therapy to treat all likely pathogens and the systemic use of corticosteroids [4].

Critical COVID-19 representing 5\% of global cases, defined by acute respiratory distress syndrome (ARDS), sepsis, septic shock or other conditions that would normally require the provision of life-sustaining therapies such as mechanical ventilation (invasive or non-invasive) or vasopressor therapy for hypotension; WHO strongly recommends the systemic use of corticosteroids in addition to Oxygen therapy (Ventilation), crystalloid fluids for resuscitation and vasopressors to maintain mean arterial pressure (MAP) $\geq$ $65 \mathrm{mmHg}[4]$.

No specific pharmaceutical products have yet been shown to be safe and effective for the wide treatment of COVID-19. However, a number of licensed medicines have been suggested as potential investigational therapies to undergo clinical trials, including the Solidarity trial co-sponsored by WHO and participating countries [6]. Investigational anti-COVID-19 therapeutics should be used only in approved, randomized controlled trials [7].

Alignment of country protocols to the international standards particularly to WHO living guidelines for clinical management of COVID-19, represent an important contribution to quality of care in WHO African countries and subsequent reduction of the case fatality rate. The WHO regional office collected and analyzed country protocols to check their consistency with the WHO guidelines on therapeutics treatment.

The purpose of this paper is to summarize the key findings of the survey including commonalities between the protocols in the WHO African region and the discrepancies vis-à-vis WHO guidelines. It provides a critical analysis of COVID-19 therapeutic treatment in Africa, especially the risk to compromise the quality of care and makes recommendations to Member States, WHO and partners to address the identified gaps.

\section{Methods}

The data were collected from October to November 2020 across the forty-seven (47) countries of the WHO African region. Through the network of medicines advisers at WHO Country Offices (WCOs), an email was sent requesting to share the country 
treatment protocols or guidelines to treat COVID-19.

Information and records of interest received from the protocols of 30 countries were organized and transcribed into a Microsoft excel 2016 spreadsheet template. To better fit the data entry of therapeutics used by each country, the template was arranged by country and by disease severity in 4 categories (Mild, Moderate, severe and critical disease).

A comparative analysis was carried out to evaluate, identify, summarize and count the country similarities on the recommended therapeutics as well as the discrepancies with regards to the WHO recommendations. While the emphasis of the study was drawn to specific COVID-19 therapeutics candidates that have been proposed to undergo clinical trials such as: Hydroxychloroquine or Chloroquine (HCQ/CQ), antivirals products, corticosteroids, immunomodulatory products and Convalescent Plasma; attention was also placed on antibacterial products recommended for Mild and Moderate COVID-19, to check the compliance with WHO recommendation against their use, except in cases of clinical suspicion of infection.

Therapeutics for symptomatic treatment recommended by WHO for Mild and Moderate cases were also considered. Finally, the variables reported and calculated were the number of countries counted for each recommended therapeutic per disease severity, then translated into percentages and displayed as graphs for easy understanding and interpretation.

Dosage forms and posology were not assessed; accent was placed on the denomination of the therapeutics recorded into the protocols. Focus was mainly made on general treatment for adults; Vulnerable populations or specific cases such as children, pregnant women, older people and peoples with underlying conditions were not reported aside.

Data analysis was only based on written information and records from the protocols, no additional information was asked and received from countries. Analysis was limited by some missing information for severe and critical disease, sometime mixed in one phase or not well described for some country protocols. Moreover, for some protocols, therapeutic class was indicated instead of the exact name of the product.

\section{Results}

Thirty (30) countries out of 47 (64\%) responded and shared their protocols by email: Algeria, Angola, Benin, Burkina Faso, Burundi, Cameroon, Central African Republic, Chad, Congo, Cote d'Ivoire, Democratic Republic of Congo, Eritrea, Gabon, Ghana, Guinea, Guinea-Bissau, Kenya, Madagascar, Mali, Mauritius, Mozambique, Namibia, Niger, Nigeria, Rwanda, Seychelles, South Africa, South Sudan, United Republic of Tanzania and Zambia.

The main findings from the analysis conducted on 30 protocols, highlight the therapeutics recommended by WHO African countries to respond to COVID-19, displayed by disease severity in four different figures and tables. Figures display the percentage of countries per recommended therapeutics for the treatment of COVID-19 by disease severity, while tables highlight the therapeutics recommended by country names and by disease severity.

Figures 1 highlights the percentage of countries per recommended therapeutics to treat mild COVID-19, whilst Table 1 shows the therapeutics recommended by countries to treat the same disease category. Fifty-three percent (53\%) of countries recommend the use of anti-bacterial (AB) products; whereas $50 \%$ of countries adopted the use of HCQ/CQ. Forty-seven percent (47\%) of countries approved symptomatic treatment, whereas $17 \%$ opted to associate Zinc in the treatment. Thirteen (13\%) approved the regimen with LPV/r that can replace HCQ/CQ for some countries in case of allergy, and only $3 \%$ validated Remdesivir, and the same percentage of countries recommended Interferon Alfa-2 Beta. 
Table 1

Therapeutics recommended by countries for the treatment of mild COVID-19.

\begin{tabular}{|c|c|c|c|c|c|c|c|}
\hline & \multicolumn{7}{|c|}{ Recommended Therapeutics } \\
\hline & $\begin{array}{l}\text { Antibacterial } \\
\text { Products }\end{array}$ & HCQICQ & $\begin{array}{l}\text { Symptomatic } \\
\text { treatment }\end{array}$ & Ziinc & Lopinavir/Ritonavir & Remdesivir & $\begin{array}{l}\text { Interferon } \\
\text { Alpha- } \\
\text { 2beta }\end{array}$ \\
\hline \multirow[t]{16}{*}{ Countries } & Algeria & Algeria & Angola & Benin & Algeria & Ghana & Gabon \\
\hline & Benin & Benin & Cote d'Ivoire & Burundi & CAR & & \\
\hline & Burkina Faso & $\begin{array}{l}\text { Burkina } \\
\text { Faso }\end{array}$ & Eritrea & Cameroun & Congo & & \\
\hline & Burundi & Burundi & Gabon & Chad & Mali & & \\
\hline & Cameroun & Cameroun & Kenya & Guinea & & & \\
\hline & CAR & CAR & Maurithius & & & & \\
\hline & Chad & Chad & Mozambique & & & & \\
\hline & Congo & Congo & Namibia & & & & \\
\hline & DRC & DRC & Nigeria & & & & \\
\hline & Ghana & Ghana & Seyschelle & & & & \\
\hline & $\begin{array}{l}\text { Guinea } \\
\text { Bissau }\end{array}$ & Guinea & South Africa & & & & \\
\hline & Guinea Rep & $\begin{array}{l}\text { Guinea } \\
\text { Bissau }\end{array}$ & South Sodan & & & & \\
\hline & Madagascar & Madagascar & Tanzania & & & & \\
\hline & Mali & Mali & Zambia & & & & \\
\hline & Niger & Niger & & & & & \\
\hline & Rwanda & & & & & & \\
\hline Total & 16 & 15 & 14 & 5 & 4 & 1 & 1 \\
\hline
\end{tabular}

Figure 2 shows the percentages of countries per recommended therapeutics to treat moderate COVID-19, whereas Table 2 indicates the therapeutics recommended by countries to treat the same cases. Twenty-four of the 30 countries $(80 \%)$ recommend the use of $A B$ products. Fifty-seven percent (57\%) of the countries adopted the use of HCQ/CQ. Twenty-three percent (23\%) authorized LPV/r that can replace HCQ/CQ in case of allergy, $20 \%$ of countries approved symptomatic treatment and the same percentage opted to associate Zinc in the treatment, $10 \%$ validated Remdesivir whereas $7 \%$ recommended Interferon Alfa-2Beta. 
Table 2

Therapeutics recommended by countries for the treatment of moderate COVID-19.

\begin{tabular}{|c|c|c|c|c|c|c|c|}
\hline & \multicolumn{7}{|c|}{ Recommended Therapeutics } \\
\hline & $\begin{array}{l}\text { Antibacterial } \\
\text { Products }\end{array}$ & HCQ/HQ & Lopinavir/Ritonavir & $\begin{array}{l}\text { Symptomatic } \\
\text { treatment }\end{array}$ & Zinc & Remdesivir & $\begin{array}{l}\text { Interferon } \\
\text { Alpha- } \\
\text { 2beta }\end{array}$ \\
\hline \multirow[t]{24}{*}{ Countries } & Algeria & Algeria & Algeria & Cote d'Ivoire & Benin & Congo & Gabon \\
\hline & Angola & Benin & CAR & Kenya & Burundi & Ghana & Eritrea \\
\hline & Benin & $\begin{array}{l}\text { Burkina } \\
\text { Faso }\end{array}$ & Congo & Maurithius & Cameroun & Namibia & \\
\hline & Burkina Faso & Burundi & DRC & Namibia & CAR & & \\
\hline & Burundi & Cameroun & Eritrea & Nigeria & Chad & & \\
\hline & Cameroun & CAR & Gabon & South Africa & Guinea & & \\
\hline & CAR & Chad & Mali & & & & \\
\hline & Chad & Congo & & & & & \\
\hline & Congo & DRC & & & & & \\
\hline & DRC & Eritrea & & & & & \\
\hline & Eritrea & Gabon & & & & & \\
\hline & Gabon & Ghana & & & & & \\
\hline & Ghana & Guinea & & & & & \\
\hline & Guinea & $\begin{array}{l}\text { Guinea } \\
\text { Bissau }\end{array}$ & & & & & \\
\hline & Guinea Bissau & Madagascar & & & & & \\
\hline & Madagascar & Mali & & & & & \\
\hline & Mali & Niger & & & & & \\
\hline & Mozambique & & & & & & \\
\hline & Niger & & & & & & \\
\hline & Rwanda & & & & & & \\
\hline & Seychelles & & & & & & \\
\hline & South Soudan & & & & & & \\
\hline & Tanzania & & & & & & \\
\hline & Zambia & & & & & & \\
\hline Total & 24 & 17 & 7 & 6 & 6 & 3 & 2 \\
\hline
\end{tabular}

Figure 3 displays the Percentage of countries per recommended therapeutics to treat severe COVID-19, while the Table 3 indicates therapeutics recommended by countries for the same disease. Fifty-three percent (53\%) of the countries recommended the use of HCQ/CQ, 47\% endorsed corticosteroids therapy, $27 \%$ agreed to use Remdesivir, $23 \%$ opted for LPV/r while $10 \%$ validated Convalescent Plasma therapy. 
Table 3

Therapeutics recommended by countries for the treatment of severe cases of COVID-19

\begin{tabular}{|c|c|c|c|c|c|}
\hline & \multicolumn{5}{|c|}{ Recommended Therapeutics } \\
\hline & $\mathrm{HCQ} / \mathrm{CQ}$ & Corticosteroids & Remdesivir & Lopinavir/Ritonavir & Convalescent Plasma \\
\hline \multirow[t]{16}{*}{ Countries } & Algeria & Burkina Faso & Congo & Algeria & Ghana \\
\hline & Burkina Faso & Algeria & Cote d'Ivoire & Congo & Maurithius \\
\hline & Burundi & Angola & DRC & Cote d'Ivoire & Rwanda \\
\hline & Cameroun & CAR & Gabon & DRC & \\
\hline & CAR & Chad & Mauritius & Gabon & \\
\hline & Chad & Congo & Namibia & Guine & \\
\hline & Congo & Ghana & Rwanda & Mali & \\
\hline & Cote d'Ivoire & Mauritius & South Africa & & \\
\hline & DRC & Mozambique & & & \\
\hline & Gabon & Namibia & & & \\
\hline & Ghana & Nigeria & & & \\
\hline & Guine & Rwanda & & & \\
\hline & Guinea Bissau & Seyschelles & & & \\
\hline & Kenya & South Africa & & & \\
\hline & Mali & & & & \\
\hline & Niger & & & & \\
\hline TOTAL & 16 & 14 & 8 & 7 & 3 \\
\hline
\end{tabular}

Figure 4 presents the percentage of countries per recommended therapeutics to treat critical COVID-19, whilst Table 4 displays Therapeutics recommended by countries for the treatment of Critical cases. Sixty percent $(60 \%)$ of the countries recommended corticosteroids, 30\% approved HCQ/CQ, 27\% validated Remdesivir, $17 \%$ authorized LPV/r, $10 \%$ validated Tocilizumab whereas $7 \%$ recommended convalescent plasma therapy. 
Table 4

Therapeutics recommended by countries for the treatment of Critical COVID-19 cases

\begin{tabular}{|c|c|c|c|c|c|c|c|}
\hline & \multicolumn{7}{|c|}{ Recommended Therapeutics } \\
\hline & Corticosteroids & $\mathrm{HCQ} / \mathrm{CQ}$ & Remdesivir & Lopinavir/Ritonavir & $\begin{array}{l}\text { Not } \\
\text { Described }\end{array}$ & $\begin{array}{l}\text { Convalescent } \\
\text { Plasma }\end{array}$ & Tocilizumab \\
\hline \multirow[t]{18}{*}{ Countries } & Angola & Algeria & $\begin{array}{l}\text { Cote } \\
\text { d'Ivoire }\end{array}$ & Algeria & Benin & Ghana & Angola \\
\hline & Algeria & Chad & Congo & Cote d'Ivoire & burkinafaso & Mauritius & Ghana \\
\hline & CAR & $\begin{array}{l}\text { Cote } \\
\text { d'Ivoire }\end{array}$ & DRC & DRC & Burundi & Rwanda & Mauritius \\
\hline & Chad & DRC & Ghana & Guine & Cameroun & & \\
\hline & Congo & Ghana & Mauritius & Mali & Chad & & \\
\hline & Eritrea & Guine & Namibia & & Gabon & & \\
\hline & Ghana & $\begin{array}{l}\text { Guine } \\
\text { Bissau }\end{array}$ & Rwanda & & & & \\
\hline & Guine & Mali & $\begin{array}{l}\text { South } \\
\text { Africa }\end{array}$ & & & & \\
\hline & Guine Bissau & Niger & & & & & \\
\hline & Madagascar & & & & & & \\
\hline & Mali & & & & & & \\
\hline & Mauritius & & & & & & \\
\hline & Mozambique & & & & & & \\
\hline & Namibia & & & & & & \\
\hline & Nigeria & & & & & & \\
\hline & Rwanda & & & & & & \\
\hline & Seyschelle & & & & & & \\
\hline & South Africa & & & & & & \\
\hline TOTAL & 18 & 9 & 8 & 5 & 5 & 3 & 3 \\
\hline
\end{tabular}

\section{Discussion}

\section{Commonalities between country protocols and discrepancies vis-à-vis the WHO recommendations}

According to the crosscutting analysis conducted on 30 country protocols used to treat COVID-19 in the WHO African region, even though WHO African countries adopted and adapted protocols based on their context, the findings demonstrated some commonalities between countries. The assessed protocols present treatments by disease severity, and most of recommended specific therapeutics were candidates originally proposed to undergo clinical trial for COVID-19 treatment. Basically, for severe and critical COVID-19, in compliance with WHO recommendations, where described all countries recommend oxygen therapy to increase and maintain $\mathrm{SpO} 2>90 \%$, vasopressors products to treat hypotension during septic shock, anticoagulant to prevent and treat venous or arterial thromboembolism, crystalloids fluids for hydration and resuscitation and AB therapy to kill all likely pathogens. 
However, significant discrepancies were noticed, when compared therapeutics recommended in country protocols against the current WHO recommendations published in the tools designed to guide clinicians' practices and policy makers. The difference resides in whether the country protocols still recommending therapeutics already dropped from Solidarity clinical trials after proving the potential harms would outweigh the potential benefices, other therapeutics are validated to be used in the nonrecommended phase of the disease or just some analyzed protocols do not validate therapeutics strongly recommended by WHO.

Considering the presented data in both figures and tables (1,2,3 and 4); HCQ/CQ has shown to be largely approved by WHO African region, because $50 \%$ of assessed country protocols recommend HCQ/CQ to treat mild COVID- 19 disease, $57 \%$ to treat moderate disease, $53 \%$ to treat severe disease and $27 \%$ to deal with critical COVID-19 disease. As established in prior conducted studies, HCQ/CQ previously described as a potent inhibitor of most coronaviruses including SARS-CoV-1 [8, 9, 10], were promoted since the beginning of the pandemic in China to demonstrate the potential broad-spectrum antiviral activity [11, 12], and immunomodulatory effects $[12,13]$. They have been proposed to undergo investigation for their possible effect against SARSCoV-2 in clinical trial [19], including solidarity trial [14, 15]. In some countries such as United States of America, the Food and Drug Administration (FDA) issued an Emergency Use Authorization (EUA) for the use of hydroxychloroquine and chloroquine to treat COVID-19 in hospitalized patients later revoked 15 June $2020[10,16]$.

Within Africa, the ANTICOV trial, which is the largest clinical trial in Africa to treat COVID-19 cases before they become severe, brought together African scientific leaders and enrolled 13 African countries in the trial to test the same products [17], HCQ/CQ were considered to be the standard of care for COVID-19 disease in numerous African countries. Likewise, the Economic Community of West African States (ECOWAS) has approved the use of chloroquine to treat coronavirus patients. ECOWAS supports the supplementary anti-viral treatment of coronavirus with hydroxychloroquine should be for mild forms of the virus [8]. The overall findings on HCQ/CQ use are consistent with the previous review study on off-label use HCQ/CQ to treat COVID-19 in Africa, which confirmed that many African countries have already approved at the national level, the use of these drugs opposing WHO warnings [18]. WHO also reported the wide use of hydroxychloroquine with Azithromycin outside of COVID-19 clinical trials, as potential threats that could affect antimicrobial stewardship activities and drive antimicrobial resistance [19].

According to WHO recommendations, HCQ trial arm has been discontinued in the solidarity trial since 17 June 2020, because it has not shown results on reduction of mortality when it can be associated with cardiotoxicity risks [20]. On 17 December 2020, an international Guideline Development Group (GDG) made a Strong recommendation against the use of HCQ/CQ in patients with COVID-19, regardless of disease severity [21]. Based on the up-to-date evidence available and aligning with WHO recommendations; many others published large scale studies concluded on balanced benefits against risks that might occur when treating COVID-19 with HCQ/CQ, worsened by Azithromycin association [22]. Even though HCQ/CQ may exhibit antiviral and immunomodulatory activities, there is no clinical evidence to support their use in WHO African Countries, their cardiovascular toxicity risks outweigh the targeted benefices $[12,16]$, they might increase the risk of lethal arrhythmias associated with QT interval prolongation $[23,24,25]$. However, a worldwide controversy about the use hydroxychloroquine and chloroquine for COVID 19 treatment was noticed, few studies reported HCQ/CQ likely to lower death rates, and advocate to encourage it use worldwide that might require further studies $[17,26]$.

On the other hand, the data pointed out the extensive validation of $A B$ products at the earliest stage of the disease, around $53 \%$ and $80 \%$ of analyzed protocols recommend the regimen with $A B$ products including Azithromycin at mild and moderate level respectively, for suspected co-infections. Similar findings on the increased rates of antimicrobial prescription for COVID-19 patients were established throughout different published studies, that could further worsen the global burden of antimicrobial resistance (AMR), especially in Africa where health systems are weak [27, 28]. The situation could be exacerbated by Azithromycin widely combined with HCQ/QC to treat COVID-19 $[19,29]$. Tackling identified antibiotic resistance in all part of the world remains a high priority for WHO, the world urgently needs to change the way it prescribes and uses antibiotics [30]. Few countries in the WHO African region have implemented the WHO Policy Package to combat antimicrobial resistance (AMR) and have national AMR plans in place [31,32]. The situation requires strong measures to fight against AMR in the pandemic era, strengthen and enforce existing antibiotic use regulations and revise existing protocols [32,33]. Antibiotics should mostly be reserved for those suffering from severe and critical COVID-19 [27, 34].

Page $9 / 19$ 
The current WHO clinical guidance does not recommend AB therapy for mild or moderate COVID-19 unless bacterial infections exist [4, 5]. From a recent review, only few patients with COVID-19 experience a secondary bacterial or fungal infection [4, 28, 35], there is no strong evidences to support the association with respiratory bacterial or fungal co-infection [36]. Ideally, blood culture would be recommended prior to the initiation of antimicrobial therapy even in severe cases, to avoid short-term side effects of $A B$ products in patients and negative long-term consequences of increased antimicrobial resistance, associated with morbidity and mortality increase, health care costs as well as the economic burden on families and societies [4, 27].

Centered to the results analysis, the antiviral Remdesivir was also counted among the commonly recommended therapeutics in the WHO African region. Twenty-seven percent (27\%) of assessed country protocols recommend Remdesivir to treat severe and critical COVID-19. Remdesivir, was proposed early among antivirals to be repurposed in the clinical trial including solidarity trial for COVID 19 treatment $[15,37,38]$. Many conducted clinical trial have not shown interesting results for its proper recommendation and approval in the treatment of COVID-19 [15,38,39]. Although until today evidence is not enough to support Remdesivir as an effective therapeutic for COVID-19 patients, this antiviral is one of the reported therapeutics under use in different countries and has been granted an EUA in some countries such as United States of America (USA) [40, 41, 63], and endorsed in the National Health Institute (NHI) treatment guidelines [42]. Further studies are needed to fully assess Remdesivir's role in COVID-19 treatment.

As per WHO clinical Guidance, Remdesivir is not recommended to be administered as a treatment or prophylaxis for COVID-19 outside the context of clinical trials. Moreover, on 20 November 2020, a conditional recommendation against the use of Remdesivir in hospitalized patients regardless the disease severity, was expressed in the WHO living guide by the international Guideline Development Group (GDG) [43].

The antiviral Lopinavir / Ritonavir (LPV/r) is also validated within the WHO African region to treat COVID-19. Thirteen percent (13\%) of analyzed protocols validate LPV/r to treat Mild disease, $23 \%$ for Moderate and Severe disease and $17 \%$ to treat critical COVID-19. LPV/r was among the potential antivirals registered in the Chinese COVID-19 Diagnosis and Treatment Plan, to be repurposed for COVID-19 treatment. It has been recommended as a first-line or second-line in many countries [15, 44]. However, based on the preliminary results made public on 29 June 2020, LPV/r did not improve clinical outcomes for hospitalized patients, whether in monotherapy or combined with interferon [45], while the overall benefit-risk profile may not be positive [42, 46, 47]. LPV/r has been dropped from solidarity clinical trial since 4 July 2020 [14]. On 17 December 2020, an international Guideline Development Group (GDG) made a Strong recommendation against the use of LPV/r in patients with COVID-19, regardless of disease severity [21]. In addition to gastrointestinal side effects reported [4], both Lopinavir and Ritonavir might be associated with hepatotoxicity, lower bone mineral density, severe bleeding in patients with hemophilia, the decrease in High Density Lipoprotein (HDL) and the Increase in Low Density Lipoprotein (LDL) [48].

Pursuant to the survey findings, corticosteroids are also generally used in Africa to treat severe and critical disease. About $47 \%$ of countries recommend corticosteroids for severe disease, while $60 \%$ of assessed protocols indicate the same products to respond to critical disease. They act by mitigating the immune response reaction and reduce cytokine formation. Most guidelines produced early in the covid-19 pandemic strongly discouraged the use of steroids for covid-19. However, the detail with which this recommendation was made varied widely. The use of steroids in acute respiratory infections such as covid-19 was originally contested, but the debate was complex and relies on the interpretation of observational studies and surrogate outcomes [49].

Nevertheless, WHO strongly recommends systemic corticosteroid therapy for the treatment of all patients with severe and critical COVID-19 [4, 50]. On the 2nd September 2020, WHO published a guidance for clinicians and health care decision-makers with a strong recommendation on the systemic use of corticosteroids, for the treatment of all patients with severe and critical COVID-19, and a conditional recommendation against systemic corticosteroids in patients with non-severe COVID-19 [51]. Many other studies established the efficacy of corticosteroids in hospitalized patients who require supplemental oxygen, with the greatest effect observed in patients who require ventilation $[42,52,53]$. The product is not recommended out of hospital treatment and for patients who do not require oxygen [54].

The use of Tocilizumab and Interferon rarely endorsed in the analyzed protocols were also among potential therapeutics proposed to undergo clinical trial for COVID 19 treatment. However, they are not recommended by WHO for the treatment or

Page 10/19 
prophylaxis of COVID-19, outside the context of clinical trials. As per available evidence, Interferon has no effect on the overall mortality, initiation of ventilation and the duration of hospital stay in hospitalized patients. Nevertheless, important side-effects have been described while their use $[4,55]$. In the other hand, some studies concluded Tocilizumab not effective for preventing intubation or death in hospitalized patients [56], while in others studies Tociluzimab was reputed to calm the inflammatory storm and reduce mortality in severe patients with COVID-19. Some countries endorsed the same products in their protocols [42,57]. Tociluzumab was among potential therapeutics formally included in the diagnosis and treatment program of COVID-19 of the national health commission of China since 3 March 2020 [57].

Even though a controversy may be noticed for some therapeutics, until there is sufficient evidence, WHO cautions against physicians and medical associations recommending or administering unproven treatments to patients with COVID-19 [14]. During times of crisis, transparent guidelines from the World Health Organization may be the only source of direction available to clinicians globally. They may be adopted internationally with only minor local adaptations [58, 59].

\section{Impact on the quality of care, the procurement and the supply chain, for the continuity of essential health services}

The need to redirect supplies to treat patients with COVID-19, compounded by general supply chain disruptions because of the outbreak on other sectors, is likely to lead to stock-outs of resources needed for essential services. Supply is dynamic during a pandemic and there are elevated risks of shortages [60].

Country treatment protocols and guidelines are important tools for effective use of therapeutics to provide optimal therapy and contribute efficiently to the country's economy. The use of non-recommended therapeutics is an unnecessary treatment likely to cause the resources wastages, the products shortage and stock out, increase of the products prices, or sometime induce substandard and falsified products as well as unnecessary stockpiling, that can lead to the supply chain and supplies management disruption, without counting probable harms on people's health. Studies reported HCQ /CQ stock piling and shortages in some African countries such as South Africa, while falsified and substandard CQ emerged in Cameroon and the Democratic Republic of Congo [18,61], poisoning and toxic effects reported in Nigeria [62], with increased market prices.

Drug supply usually base on standards treatment protocols and guidelines especially in countries with limited resources; the use of inappropriate therapeutics may misguide the procurement and forecasting process where unnecessary products may be largely procured on the detriment of essential products to respond to the disease, without counting probable harms on people's health that misused medicines might cause.

Throughout the conducted survey, the identified prescription of medicines for off-label use may be subject to national laws and regulations. All health care workers in member states should be aware of and comply with the laws and regulations governing their practice in COVID-19 response [6]. International partners as well as key players involved in COVID-19 response would play a strategic role, by procuring and availing only relevant products in the COVID-19 context.

Even though updating protocols timely may be difficult especially during the pandemic outbreak, where information are changing and updated time to time when new scientific knowledge emerges; it is the utmost way to ensure appropriate therapy and overcome the pandemic burden, especially in WHO African region with limited resources.

\section{Conclusion}

During the novel public health emergency of international concern, strong collaboration, solidarity, network spirit and sharing reliable proven tools to guide practices, are imperative way to overcome the unprecedented COVID-19 outbreak burden, especially when the large-scale uncertainty exists. Alignment of country protocols to the international standards particularly to WHO guidelines for clinical management of COVID-19, represent an important contribution to the health care outcome and reduction of the case fatality rate.

The survey carried out by WHO regional office for Africa to analyze countries' protocols established some commonalities across WHO African countries, but also pointed out significant deviation from WHO recommendations in response to COVID-19, as reported by many other conducted studies. Despite the efforts demonstrated by countries to respond to the pandemic, some 
countries still recommend specific therapeutics discontinued in the clinical trials while others do not validate the strongly recommended lifesaving therapeutics in their protocols. Moreover, this analysis revealed a possible AB products misuse that might impact on antimicrobial resistance surge. Regional interventions and national sustainable strategies are imperative to address the identified gaps. In the context of COVID-19, the use of timely updated protocols in all countries would be essential to ensure optimal therapy, decrease the case fatality rate and reduce any likely impact on Procurement and Supply Management. Treatment protocols developed during emergency, would be taken as living tools subject to regular review.

\section{Recommendations}

WHO and Partners should :( a) Raise awareness and support Member States to timely update and implement new clinical recommendations, in line with evolving scientific evidences; (b) Assess Member States perceptions and reasons underpinning the implementation of WHO recommendations for more impactful support to countries; (c) Enhance risk mitigation to tackle AMR surge in the WHO African region.

Member States should: (a) Proactively revise the protocols according to new evidence; (b) Strengthen the capacity of health workers and keep them abreast with trends and innovation in clinical management of COVID-19 to ensure the quality of care; (c) Reinforce the optimal use of COVID 19 therapeutics to prevent AMR surge; (d) Strengthen networking for sharing best practices and experience on clinical management of COVID-19.

\section{List Of Abbreviations}

$\mathrm{AB} \quad$ Antibacterial

ACT-A Access to COVID-19 Tool Accelerator

AMR Antimicrobial resistance

ARDS Acute respiratory distress syndrome

COVID-19 Corona virus disease 2019

CQ Chloroquine

ECOWAS Economic Community of West African States

EUA Emergency Use Authorization

FDA Food and Drug Administration

GDG Guideline Development Group

HCQ Hydroxychloroquine

HDL High Density Lipoprotein (HDL)

ICU Intensive Care Unit

IMST Incident management support team

LDL Low Density Lipoprotein (LDL)[

LPV/r Lopinavir / Ritonavir (LPV/r)

MAP Mean arterial pressure

$\mathrm{NHI}$ National Health Institute (NHI) 
SAGE Strategic Advisory Group of Experts

SARS-COV-2 severe acute respiratory syndrome coronavirus-2

SPO2 Oxygen saturation

SRD Severe respiratory distress

USA United States of America

WCO WHO Country Offices

WHO World Health Organization

\section{Declarations}

\section{Ethics approval and consent to participate}

Not applicable, since this research does not include human participants, no permission was needed from the WHO African Region Ethics Review Committee.

\section{Consent for publication}

Not applicable as manuscript does not include details, images or videos relating to individual persons.

\section{Availability of data and material}

The datasets generated and/or analyzed during the current study are not publicly available, but they are available from the corresponding author on reasonable request.

\section{Competing interest}

The authors declare that they have no competing interests.

\section{Funding:}

The study was supported by unrestricted program funds of the WHO Regional Office for Africa, Universal Health Coverage/Life Course Department. The WHO staff involved in this study are listed as co-authors to the paper and their roles were as follows, JBN who is the Team Leader for the Medicines, Health Infrastructures and Equipment maintenance program, provided technical guidance for the study and with OMJK contributed to the study conception and design, and accompanied IM, the lead author. EA, SK, AL, AS, DT and RM are members of the Medicines, Health Infrastructures and Equipment maintenance (MIM) team. They read different versions of the manuscript and provided valuable contributions and inputs to the manuscript. BG is the Director of the Universal Health Coverage/Life Course Cluster under whose leadership the Medicines, Health Infrastructures and Equipment maintenance program falls. BG provided the overall leadership and guidance for the study.

\section{Authors' contributions}

MIM team contributed to the study conception and design, data collection, data analysis and writing of the manuscript. JBN and OMJK and EA contributed on the paper design; considerably reviewed the paper and provided substantial contribution on data interpretation. SK, AL, AS, DT, RM and BG provided inputs in the analysis of the manuscript. All authors read and approved the final manuscript.

\section{Acknowledgement}


We are thankful to the Medicine Advisors at WHO Country Offices (WCOs) who facilitated in country protocols collection. However, the article contains the insights and views of the authors only.

\section{References}

1. WHO. Timeline: WHO's COVID-19 response. World Health Organization. 2021. Available from: https://www.who.int/emergencies/diseases/novel-coronavirus-2019/interactive-timeline? gclid=Cj0KCQiApY6BBhCsARIsAOI_GjZWOVKyv_k95eiG9s8X_x5kAVJMMIUbi7PQ38dCVjkWEIPqw5HP18aAugdEALw_wcB\#event-206. Accessed on 12 February 2021.

2. WHO. Coronavirus disease 2019 (COVID-19). Situation report 100. World Health Organization, Geneva April 29, 2020. Available from: https://www.who.int/docs/default-source/coronaviruse/situation-reports/20200429-sitrep-100-covid-19.pdf? sfvrsn=bbfbf3d1_6. Accessed: 17 March 2020.

3. WHO. A guide to WHO's guidance on COVID-19, 17 July 2020. World Health Organization; 2020. Available from: https://www.who.int/news-room/feature-stories/detail/a-guide-to-who-s-guidance. Accessed on 10 January 2021.

4. WHO. Clinical management of COVID-19, 27 May 2020. World Health Organization; 2020. Available from: https://www.who.int/publications/i/item/clinical-management-of-covid-19. Accessed 17 November 2020.

5. WHO. COVID-19 Clinical management, Living Guidance, 25 January 2021. World Health Organization; 2021. Available from: https://apps.who.int/iris/bitstream/handle/10665/338882/WHO-2019-nCoV-clinical-2021.1-eng.pdf? sequence=1\&isAllowed=y.Accessed on 11 February 2021.

6. WHO. Off-label use of medicines for COVID-19, scientific brief, 31 March 2020. World Health Organization; 2020. Available from: https://www.who.int/publications/i/item/off-label-use-of-medicines-for-covid-19-scientific-brief. Accessed on January 16, 2021.

7. WHO. Clinical care of severe acute respiratory infections - Tool kit. 11 April 2020. World Health Organization; 2020. Available from: https://www.who.int/publications/i/item/clinical-care-of-severe-acute-respiratory-infections-tool-kit. Accessed on 12 January 2021.

8. Devaux CA, Rolain J-M, Colson P, Raoult D. New insights on the antiviral effects of chloroquine against coronavirus: what to expect for COVID-19? (2020) Int J Antimicrob Agents 105938:105938. Available from: https://doi.org/10.1016/j.ijantimicag.2020.105938

9. Touret F, de Lamballerie X, 2020. Of chloroquine and COVID-19. Antiviral Res 177: 104762. DOI: 10.1016/j.antiviral.2020.104762. Available from: https://pubmed.ncbi.nlm.nih.gov/32147496/. Accessed 12 March 2021.

10. Christine Cassidy, Danielle Dever, Laura Stanbery, Gerald Edelman, Lance Dworkin \& John Nemunaitis (2020). FDA efficiency for approval process of COVID-19 therapeutics. Available from:

https://infectagentscancer.biomedcentral.com/articles/10.1186/s13027-020-00338-z\#Bib1. Accessed on 13March 2021.

11. Gao J, Tian Z, Yang X (2020). Breakthrough: chloroquine phosphate has shown apparent efficacy in treatment of COVID-19 associated pneumonia in clinical studies. Biosci Trends 14:72-73. https://doi.org/10.5582/bst.2020.01047

12. Ibáñez, S., Martínez, O., Valenzuela, F. et al. Hydroxychloroquine and chloroquine in COVID-19: should they be used as standard therapy? Clin Rheumatol 39, 2461-2465 (2020). https://doi.org/10.1007/s10067-020-05202-4. Available from: https://link.springer.com/article/10.1007/s10067-020-05202-4. Accessed 12 March 2021.

13. Savarino A, Boelaert JR, Cassone A, Majori G, Cauda R (2003). Effects of chloroquine on viral infections: an old drug against today's diseases. Lancet Infect Dis 3:722-727. https://doi.org/10.1016/S1473-3099(03)00806-5.

14. WHO. Solidarity clinical trial for COVID-19 treatments. World Health Organization; 2020. Available from: https://www.who.int/emergencies/diseases/novel-coronavirus-2019/global-research-on-novel-coronavirus-2019ncov/solidarity-clinical-trial-for-covid-19-treatments. Accessed on 23 January 2021.

15. WHO Solidarity Trial Consortium: Repurposed Antiviral Drugs for Covid-19 - Interim WHO Solidarity Trial Results (2020). Available from: https://www.nejm.org/doi/full/10.1056/NEJMoa2023184\#article_citing_articles. Accessed 15 March 2021.

16. FDA. FDA Revokes Emergency Use Authorization for Chloroquine and Hydroxychloroquine. Press Announcements 2020. Available from: https://www.fda.gov/news-events/press-announcements/coronavirus-covid-19-update-fda-revokes- 
emergency-use-authorization-chloroquine. Accessed 13 March 2021

17. DNDi. Largest clinical trial in Africa to treat COVID-19 cases before they become severe is launched in 13 countries, 16 December 2020. Drugs for Neglected Disease initiative, 2020. Available from: https://dndi.org/press-releases/2020/anticovlargest-clinical-trial-africa-treat-mild-moderate-covid-19-cases-launched-13-countries/. Accessed on January 15, 2021.

18. Isaac K. Ghana. Kenya approve the use of Chloroquine to treat COVID-19 patients. Taylor and Francis group. 2020. Available from: https://africafeeds.com/2020/04/01/ghana-kenya-approve-use-of-chloroquine-to-treat-covid-19-patients/. Accessed 03 March 2021.

19. Jeremy Hsu. freelance journalist: How covid-19 is accelerating the threat of antimicrobial resistance, (2020). BMJ 2020; 369 doi: https://doi.org/10.1136/bmj.m1983 (Published 18 May 2020)

20. WHO. Coronavirus disease (COVID-19): Hydroxychloroquine, 19 June 2020. World Health Organization, 2020. Available from: https://www.who.int/news-room/q-a-detail/coronavirus-disease-covid-19-hydroxychloroquine. Accessed on 12 January 2021.

21. WHO. Therapeutics and COVID-19: living guideline, 17 December 2020. World Health Organization, 2020. Available from: https://apps.who.int/iris/handle/10665/337876. Accessed on 23 January 2021.

22. European Medicine Agency (Science Medicines Health) (2020). COVID-19: reminder of risk of serious side effects with chloroquine and hydroxychloroquine. Available from: https://www.ema.europa.eu/en/news/covid-19-reminder-risk-seriousside-effects-chloroquine-hydroxychloroquine. Accessed 13 March 2021.

23. Archana Ramireddy, Harpriya Chugh, Kyndaron Reinier et al. Experience with Hydroxychloroquine and Azithromycin in the Coronavirus Disease 2019 Pandemic: Implications for QT Interval Monitoring (2020) DOI: 10.1161/JAHA.120.017144. Available from: https://www.ncbi.nlm.nih.gov/pmc/articles/PMC7429030/.Accessed 13 March 2021.

24. Mercuro NJ, Yen CF, Shim DJ, et al. Risk of QT Interval Prolongation Associated with Use of Hydroxychloroquine with or Without Concomitant Azithromycin Among Hospitalized Patients Testing Positive for Coronavirus Disease 2019 (COVID-19). JAMA Cardiol. 2020; 5(9):1036-1041. doi:10.1001/jamacardio.2020.1834. Available from:

https://pubmed.ncbi.nlm.nih.gov/32936252/. Accessed 10 March 2021.

25. Ehud Chorin, Lalit Wadhwani, Silvia Magnani et al. QT interval prolongation and torsade de pointes in patients with COVID19 treated with hydroxychloroquine/azithromycin (2020). DOI: 10.1016/j.hrthm.2020.05.014. Available from: https://www.heartrhythmjournal.com/article/S1547-5271(20)30435-5/fulltext. Accessed 13March 2021.

26. Brian Giesbrecht. Commentary, COVID-19: Hydroxychloroquine is Widely Used Around the Globe. Available from: https://fcpp.org/2020/11/08/hydroxychloroquine-is-widely-used-around-the-globe/. Accessed on 08 March 2021.

27. Chinwe Juliana Iwu, Portia Jordan, Ishmael Festus Jaja et al. Treatment of COVID-19: implications for antimicrobial resistance in Africa (2020). DOI: 10.11604/pamj.supp.2020.35.23713. Available from:

https://pubmed.ncbi.nlm.nih.gov/33282074/. Accessed on 13 March 2021.

28. University of Minnesota. Center for Infectious Disease Research and Policy: Study adds more evidence of antibiotic overuse in COVID-19 patients (2020). Available from: https://www.cidrap.umn.edu/news-perspective/2021/03/study-adds-moreevidence-antibiotic-overuse-covid-19-patients. Accessed 14 March 2021.

29. Mehreen Arshad, Syed Faisal Mahmood, Mishal Khan, Rumina Hasan. Covid - 19, misinformation, and antimicrobial resistance, (2020). doi: https://doi.org/10.1136/bmj.m4501. Available from: https://www.bmj.com/content/371/bmj.m4501. Accessed on 15 March 2021.

30. World Health Organization. 2020 Antibiotic resistance; 2020. Available from: https://www.who.int/news-room/factsheets/detail/antibiotic-resistance. Accessed March 12, 2021.

31. S.Y. Essack, A.T. Desta, R.E. Abotsi, E.E. Agoba. Antimicrobial resistance in the WHO African region: current status and roadmap for action. Available from: https://academic.oup.com/jpubhealth/article/39/1/8/3065721. Accessed 12 March 2021.

32. Mary Richard Akpan, Nsisong Udom Isemin, Arit Esio Udoh, Diane Ashiru-Oredope. Implementation of antimicrobial stewardship programmes in African countries: a systematic literature review, (2020). DOI: 10.1016/j.jgar.2020.03.009. Available from: https://www.sciencedirect.com/science/article/pii/S2213716520300734. Accessed 16 March 2021. 
33. Africa CDC on the African Union. Call for Review of the African Antibiotic Treatment Guidelines for Common Bacterial Infections and Syndromes (2020). Available from: https://africacdc.org/download/african-antibiotic-treatment-guidelinesfor-common-bacterial-infections-and-syndromes/. Accessed 15 March 2021.

34. Jeremy Hsu. freelance journalist: How covid-19 is accelerating the threat of antimicrobial resistance, (2020). BMJ 2020; 369 doi: https://doi.org/10.1136/bmj.m1983. Published 18 May 2020.

35. World Health Organization. Record number of countries contribute data revealing disturbing rates of antimicrobial resistance; 2020. Available from: https://www.who.int/news-room/detail/01-06-2020-record-number-of-countries-contributedata-revealing-disturbing-rates-of-antimicrobial-resistance. Accessed 13 March 2021.

36. Timothy M Rawson, Luke S P Moore, Nina Zhu et al. Bacterial and Fungal Coinfection in Individuals with Coronavirus: A Rapid Review to Support COVID-19 Antimicrobial Prescribing (2020). DOI: 10.1093/cid/ciaa530. Available from: https://pubmed.ncbi.nlm.nih.gov/32358954/. Accessed 15 March 2021.

37. Wang M, Cao R, Zhang L, et al. Remdesivir and chloroquine effectively inhibit the recently emerged novel coronavirus (2019nCoV) in vitro. Cell Res 2020; 30: 269 - 71. Available from: https://www.nature.com/articles/s41422-020-0282-0. Accessed 12 March 2021.

38. Miguel Angel Martinez. Clinical Trials of Repurposed Antivirals for SARS-CoV-2 (2020). DOI: 10.1128/AAC.01101-20. Available from: https://pubmed.ncbi.nlm.nih.gov/32631826/. Accessed 12 March 2021.

39. Ambedkar Kumar Yadav, Siwan Wen, Xianghuai Xu, Li Yu. Antiviral treatment in COVID-19: which is the most promising? -a narrative review (2021). DOI: 10.21037/apm-20-1755. Available from: https://pubmed.ncbi.nlm.nih.gov/33440983/. Accessed 12 March 2021.

40. Cassidy, C., Dever, D., Stanbery, L. et al. FDA efficiency for approval process of COVID-19 therapeutics (2020). https://doi.org/10.1186/s13027-020-00338-z. Available from: https://infectagentscancer.biomedcentral.com/articles/10.1186/s13027-020-00338-z. Accessed 11 March 2021.

41. Tarik Asselah, David Durantel, Eric Pasmant et al. COVID-19: Discovery, diagnostics and drug development (2020). DOI: 10.1016/j.jhep.2020.09.031. Available from: https://pubmed.ncbi.nlm.nih.gov/33038433/. Accessed 12 March 2021.

42. COVID-19 Treatment Guidelines Panel. Coronavirus Disease 2019 (COVID-19) Treatment Guidelines. National Institutes of Health. Available from: https://www.covid19treatmentguidelines.nih.gov/. Accessed 16 March 2021.

43. WHO. Therapeutics and COVID-19: living guidelines, 20 November 2020. World Health Organization; 2020. Available from: https://apps.who.int/iris/handle/10665/336729. Accessed on 23 January 2021.

44. Bzikha Reda, Bouhmou Ayoub, Bzikha Ilham and Bouchnafati Sanaa. Journal of Antivirals \& Antiretrovirals. Remdesivir and Lopinavir/Ritonavir as Potential Drugs to Treat Corona Virus Disease 2019. Available from: https://www.longdom.org/openaccess/remdesivir-and-lopinavirritonavir-as-potential-drugs-to-treat-corona-virus-disease-2019-59152.html. Accessed 12 March 2021.

45. Peter W Horby, Marion Mafham, Jennifer L Bell et al. Lopinavir-ritonavir in patients admitted to hospital with COVID-19 (RECOVERY): a randomised, controlled, open-label, platform trial, 5 October 2020. DOI: https://doi.org/10.1016/S01406736(20)32013-4. Available from: https://www.thelancet.com/journals/lancet/article/PIIS0140-6736(20)32013-4/fulltext. Accessed 15 March 2021.

46. Vicki Osborne, Miranda Davies, Samantha Lane et al. Lopinavir-Ritonavir in the Treatment of COVID-19: A Dynamic Systematic Benefit-Risk Assessment (2020). DOI: 10.1007/s40264-020-00966-9. Available from: https://pubmed.ncbi.nlm.nih.gov/32578156/. Accessed 12 March 2021.

47. Bin Cao Bin, WangYeming et al. A Trial of Lopinavir-Ritonavir in Adults Hospitalized with Severe Covid-19 (2020). DOI: 10.1056/NEJMoa2001282. Available from: https://www.nejm.org/doi/full/10.1056/NEJMoa2001282. Accessed 12 March 2021.

48. Dragana Javorac, Lazar Grahovac, Luka Manić et al. An overview of the safety assessment of medicines currently used in the COVID-19 disease treatment 21 July 2020. doi: 10.1016/j.fct.2020.111639. Available from: https://www.ncbi.nlm.nih.gov/pmc/articles/PMC7372271/. Accessed 18 March 2021. 
49. Dagens Andrew, Louise Sigfrid et al. Scope, quality, and inclusivity of clinical guidelines produced early in the covid-19 pandemic: rapid review (2020). Available from: https://www.bmj.com/content/bmj/369/bmj.m1936.full.pdf. Accessed 12 March 2021.

50. WHO. Solidarity clinical trial for COVID-19 treatments. World Health Organization,2020. Available from: https://www.who.int/emergencies/diseases/novel-coronavirus-2019/global-research-on-novel-coronavirus-2019ncov/solidarity-clinical-trial-for-covid-19-treatments. Accessed on 23 January 2021.

51. WHO. Corticosteroids for COVID-19: Living Guidance, 2 September 2020. World Health Organization, 2020. Available from: https://www.who.int/publications/i/item/WHO-2019-nCoV-Corticosteroids-2020.1. Accessed on 12 January 2021.

52. RECOVERY Collaborative Group, Horby P, Lim WS, et al. Dexamethasone in hospitalized patients with COVID-19-preliminary report. N Engl J Med. 2020. Available from: https://www.ncbi.nlm.nih.gov/pubmed/32678530. Accessed 12 March 2021.

53. National Institutes of Health. COVID-19 Treatment Guidelines Search, Therapeutic Management of Adults with COVID-19 (2021). Available from: https://www.covid19treatmentguidelines.nih.gov/therapeutic-management. Accessed 16 March 2021.

54. Whitty C. Dexamethasone in the treatment of COVID-19: Implementation and management of supply for treatment in hospitals. London: Medicines and Healthcare Products Regulatory Agency, June 16, 2020. Available from: https://www.cas.mhra.gov.uk/ViewandAcknowledgment/ViewAlert.aspx?AlertID. Accessed 15 March 2021.

55. Sarah Shalhoub. Interferon beta-1b for COVID-19. The Lancet (2020). DOI: 10.1016/S0140-6736(20)31101-6. Available from: https://www.thelancet.com/journals/lancet/article/PIIS0140-6736(20)31101-6/fulltext. Accessed 15 March 2021.

56. John H. Stone, M.D., M.P.H., Matthew J et al. Efficacy of Tocilizumab in Patients Hospitalized with Covid-19 (December 2020). Available from: https://www.nejm.org/doi/full/10.1056/NEJMoa2028836\#article_citing_articles. Accessed 12 March 2021.

57. Binqing Fu, Xiaoling Xu \& Haiming Wei: Why tocilizumab could be an effective treatment for severe COVID-19? Available from: https://translational-medicine.biomedcentral.com/articles/10.1186/s12967-020-02339-3. Accessed 15 March 2021.

58. Andrew Dagens, Louise Sigfrid, Erhui Cai et al. Scope, quality, and inclusivity of clinical guidelines produced early in the covid-19 pandemic: rapid review (2020). Available from: https://www.bmj.com/content/369/bmj.m1936. Accessed 12 March 2020.

59. Sinclair D, Isba R, Kredo T, Zani B, Smith H, Garner P. World Health Organization guideline development: an evaluation (2013). DOI: 10.1371/journal.pone.0063715. Available from: https://pubmed.ncbi.nlm.nih.gov/23741299/. Accessed 17 March 2021.

60. WHO. Maintaining essential health services: operational guidance for the COVID-19 context: interim guidance, 1 June 2020. World Health Organization; 2020. Available from: https://apps.who.int/iris/bitstream/handle/10665/332240/WHO-2019nCoV-essential_health_services-2020.2-eng.pdf?sequence=1\&isAllowed=y. Accessed on 15 January 2021.

61. Pascale M Abena, Eric H Decloedt, Emmanuel Bottieau et al. Chloroquine and Hydroxychloroquine for the Prevention or Treatment of COVID-19 in Africa: Caution for Inappropriate Off-label Use in Healthcare Settings (2020. DOI: 10.4269/ajtmh.20-0290. Available from: https://www.ajtmh.org/view/journals/tpmd/102/6/article-p1184.xml. Accessed 13 March 2021.

62. Stephanie B, Bukola A. Nigeria records chloroquine poisoning after Trump endorses it for coronavirus treatment. CNN. 2020. Available from: https://edition.cnn.com/2020/03/23/Africa/chloroquine-trump-Nigeria-intl/index.html. Accessed 17 March 2021.

63. U.S. Department of Health and Human Services. AVAILABLE COVID-19 TREATMENT OPTIONS. Available from: https://combatcovid.hhs.gov/i-have-covid-19-now/available-covid-19-treatment-options. Accessed 19 March 2021.

\section{Figures}




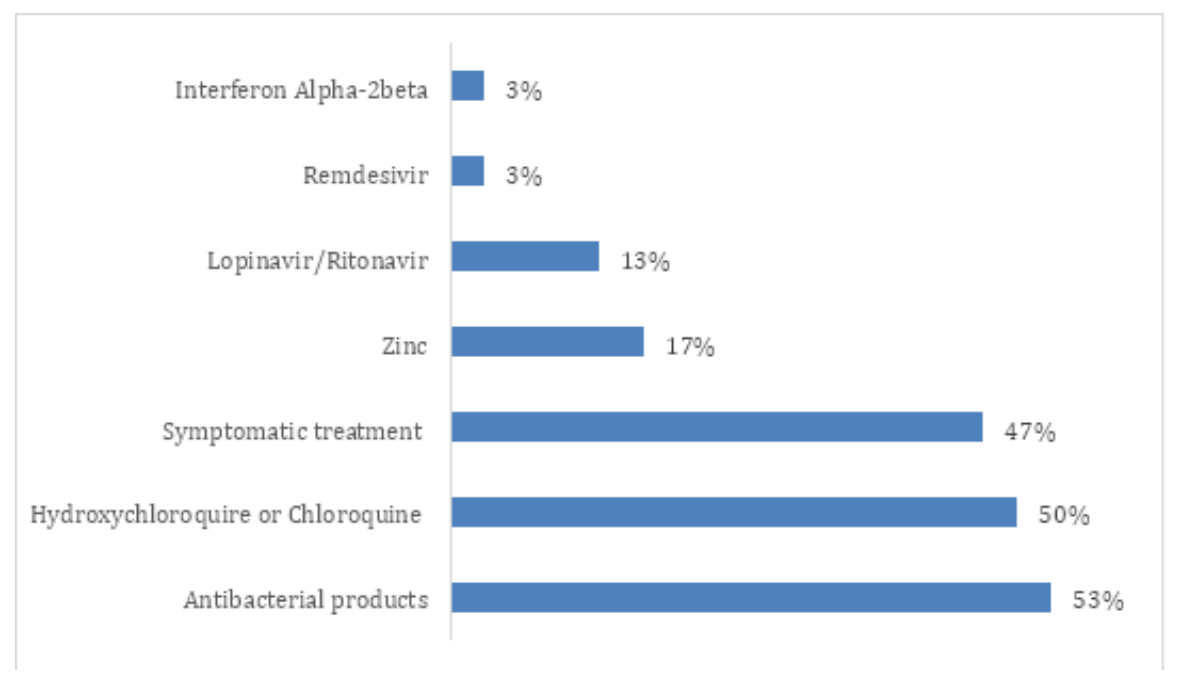

\section{Figure 1}

Percentage of countries per recommended therapeutics to treat mild COVID-19

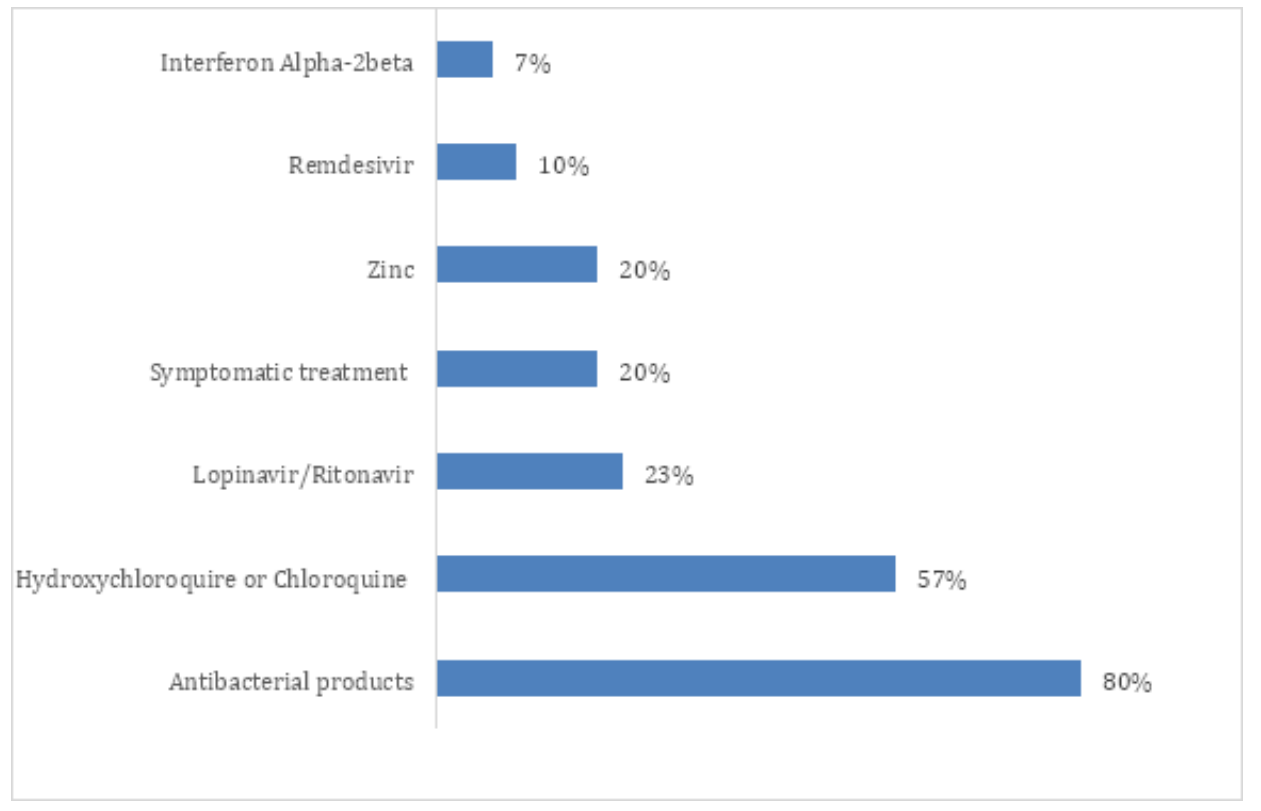

\section{Figure 2}

Percentage of countries per recommended therapeutics to treat moderate COVID-19.

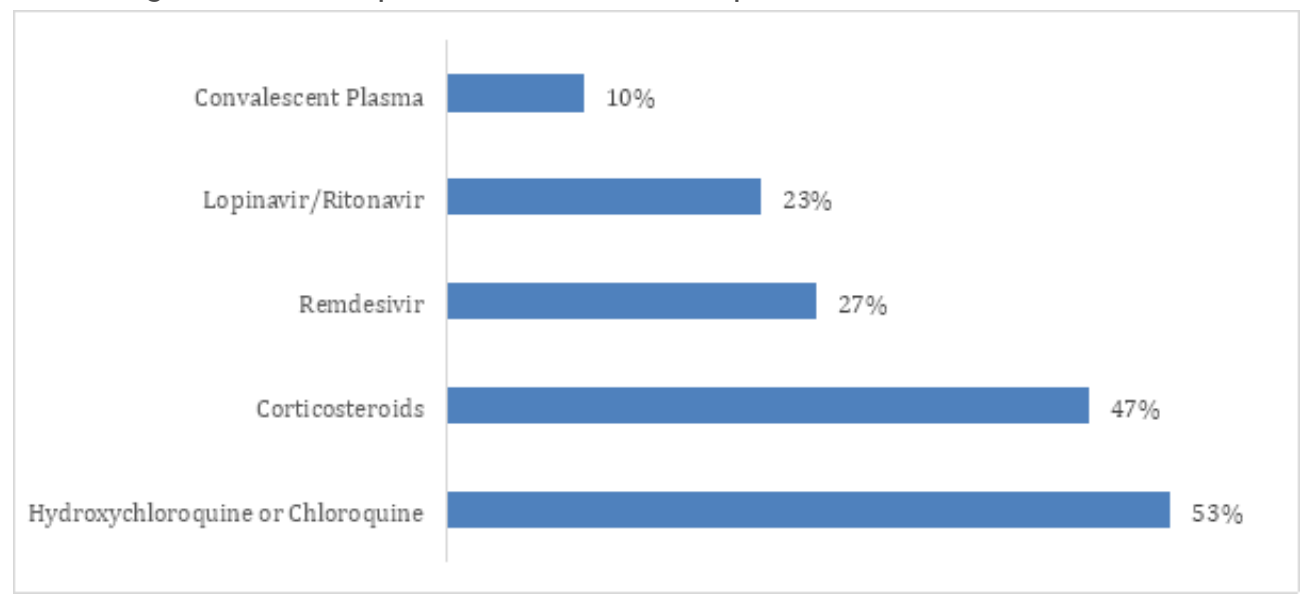

Figure 3

Page 18/19 
Percentage of countries recommended therapeutics to treat severe COVID-19

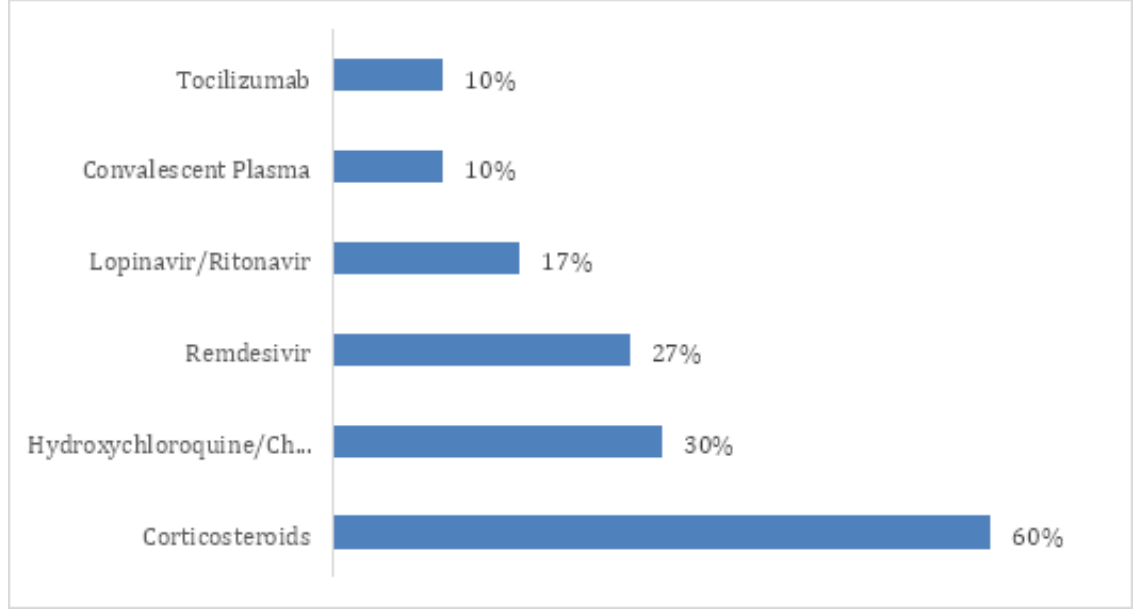

\section{Figure 4}

Percentage of countries per recommended therapeutics to treat critical COVID-19 